\title{
Representational momentum and anisotropies in nearby visual space
}

\author{
Timothy L. Hubbard $^{1} \cdot$ Susan E. Ruppel ${ }^{2}$
}

Published online: 18 October 2017

(C) The Psychonomic Society, Inc. 2017

\begin{abstract}
The possibility of anisotropies in visual space in and near the final location of a moving target was examined. Experiments 1 and 2 presented a moving target, and after the target vanished, participants indicated the final location of the leading or trailing edge of the target. Memory for both edges was displaced forward from the actual final locations, and the magnitude of displacement was smaller for the leading edge. Experiments 3 and 4 also presented stationary objects in front of and behind the final location of the target, and participants indicated the location of the nearest or farthest edge of one of the stationary objects. Memory for the near or far edge of an object in front of the target was displaced backward, and memory for the near or far edge of an object behind the target was displaced forward; the magnitude of displacement was larger for objects in front of the target and when the edge was farther away. The findings (a) suggest representational momentum is associated with an anisotropy of visual space that extends across and outward from the moving target and (b) are consistent with previous findings regarding estimation of time-to-contact, anorthoscopic perception, and memory psychophysics.
\end{abstract}

KEYWORDS Representational momentum · Anisotropy · Displacement $\cdot$ Spatial cognition

The judged final location of a recently viewed moving target is often displaced in the direction of motion, and this has been

Timothy L. Hubbard

timothyleehubbard@gmail.com

1 Arizona State University, Tempe, AZ 85281, USA

2 University of South Carolina, Upstate, Spartanburg, SC 29303, USA referred to as representational momentum (for reviews, see Hubbard, 2005, 2014a). It is not clear whether this displacement reflects a distortion in spatial representation that is limited to the target or whether the distortion also extends along the path of (previous or potential) target motion into nearby visual space. Even if effects of representational momentum are limited to a moving target and do not influence (objects in) nearby visual space, it is not clear if those effects are of an equal magnitude across all parts of that target. Given that previous studies of representational momentum typically had participants indicate either the center of the final location of the target or judge whether a probe similar in size and shape to the target was at the same location as the final location of the target, it has not generally been possible to examine the relationship between representational momentum for a moving target's location and distortions in spatial representation for different parts of the target or for different locations in nearby space. The experiments reported here examine if representational momentum is linked to anisotropies (i.e., directional differences or asymmetries) in spatial representation by measuring displacement of different parts of a moving target and displacement of different parts of stationary objects located in front of or behind the final location of the target. How these anisotropies are related to other findings regarding spatial representation is also considered.

Although studies of anisotropies in perceived space generally focus on static targets (for review, see Aznar-Casanova \& Bernardino, in press), there is evidence of anisotropies in visual space that are related to a moving target. In a study of the flash-lag effect, in which the location of a briefly presented (flashed) object aligned with the location of a moving target is perceived to lag behind that target (for review, see Hubbard, 2014b), Watanabe, Nijhawan, Khurana, and Shimojo (2001; Watanabe, 2004) found that a flashed object aligned with the leading edge of a moving target resulted in a larger flash-lag 
effect than did a flashed object aligned with the trailing edge of a moving target. Watanabe and Yokoi (2006) found that flashed objects were mislocalized toward a single point that followed the moving object at a fixed distance. Watanabe and Yokoi also found that target motion did not influence the perceived shape of a flashed object, and they suggested that information regarding the shape of an object was encoded before information regarding the location of that object was encoded (i.e., anisotropic mislocalization operated after visual object representation). Studies of Watanabe and colleagues addressed the localization and shape of a flashed object but did not address potential anisotropic distortions in the shape of the moving target or along the path (rather than just in the general direction) of motion.

Whitney and Cavanagh (2000) presented a series of studies in which flashed objects were presented near a rotating radial grating or translating linear gratings. The flashed objects were mislocalized in the direction of grating motion, and Whitney and Cavanagh concluded that the perceived location of a stationary object could be influenced by motion signals from a distant region of the visual field. ${ }^{1}$ Such mislocalizations were consistent with an influence of representational momentum from the gratings spreading to the flashed objects (cf. Hubbard, 1995; Jancke \& Erlhagen, 2010; Müsseler, Stork, \& Kerzel, 2002), but such an interpretation was not considered. The possibility of such mislocalization within a representational momentum framework was examined by Hubbard (2008), who found that memory for the location of a stationary object above or below the final location of a horizontally moving target was displaced in the direction of target motion, and this displacement decreased with increases in the distance of the stationary object from the moving target. Thus, studies of the flash-lag effect and of representational momentum suggest the presence of a moving target can influence localization of a nearby stationary object. Also, and although not involving mislocalization per se, a higher discrimination threshold for oblique motion than for vertical or horizontal motion (e.g., Ball \& Sekuler, 1980; Gros, Blake, \& Hiris, 1998) is consistent with the notion of motion-related anisotropies.

If there are anisotropies in nearby visual space that are related to target motion, then perceptual sensitivity should differ across different regions of space. Consistent with this, Kerzel, Jordan, and Müsseler (2001) presented a probe in front of or behind the final target location. The probe was a circle containing a small gap, and participants had to detect whether

\footnotetext{
${ }^{1}$ The perceived location of moving objects can also be influenced by motion signals from a distant part of the visual field. Hubbard (1993) presented a rotating target that was surrounded by a larger square frame that rotated in the same direction as the target or in the direction opposite to the target. Representational momentum of the target increased when the frame rotated in the same direction as the target and decreased when the frame rotated in the direction opposite to the target.
}

the gap was at the top or bottom of the circle. When probes were presented 100 milliseconds after the target vanished (a latency at which representational momentum can be observed; e.g., Freyd \& Johnson, 1987; Kerzel, 2000), detection of the gap was better if the probe was slightly in front of the final location of the target than if the probe was slightly behind the final location of the target. A decreased sensitivity for probes behind the target is consistent with the notion of inhibition of return, in which attention is less likely to return to a recently attended location than to move to a new location (e.g., Posner \& Cohen, 1984; Posner, Rafal, Choate, \& Vaughan, 1985); that is, locations behind the final location of the target have just been attended (as the target moved through those locations), and so participants take longer to detect a target in those locations. Complementary to inhibition of return is the notion of attentional momentum (Pratt, Spalek, \& Bradshaw, 1999; for review, see Hubbard, 2014a), which suggests that it is easier and faster to detect a target located further along the path of motion of attention than a target located an equal distance away but in a different direction.

The current experiments examined the possibility of an anisotropy in visual space that is linked with representational momentum. In Experiments 1 and 2, participants indicated the final location of the leading edge or the trailing edge of a moving target. In Experiments 3 and 4, participants indicated the location of the nearest edge or the farthest edge of a stationary object that was in front of or behind the final location of the target. It is possible that representational momentum reflects a rigid shift of the represented location of a target with no concurrent influence on the representation of the surrounding space (or on objects in the surrounding space). However, findings of Watanabe et al. (2001), Whitney and Cavanagh (2000), and Hubbard (2008) suggest that motion of the target will be linked with effects on the representation of location of nearby objects. Furthermore, findings of Kerzel et al. (2001) suggest that effects of representational momentum on the representation of nearby visual space would be asymmetrical and thus anisotropic. Given that representational momentum is in the direction of anticipated motion, there might be a contraction or compression of space between the target and an object in front of the target's location, and such compression would be consistent with findings of an underestimation of time-tocontact (e.g., Gray \& Thornton, 2001) and spatial compression prior to saccadic movement (e.g., Lappe, Awater, \& Krekelberg, 2000; Ross, Morrone, \& Burr, 1997; Sheth \& Shimojo, 2001).

\section{Experiment 1}

In Experiment 1, participants viewed a computer-animated target moving rightward, leftward, downward, or upward. After the target vanished, an auditory tone was briefly 
presented; a high tone $(2000 \mathrm{~Hz})$ cued participants to indicate the final location of the leading edge of the target, and a low tone $(250 \mathrm{~Hz})$ cued participants to indicate the final location of the trailing edge of the target. Participants indicated the location of the judged edge by positioning a cursor (via movement of a computer mouse), and any difference between the indicated location and the actual location was assessed. If representational momentum occurs and is not linked to anisotropies in the spatial representation of the target, then judgments of the final location of the leading edge of the target and the final location of the trailing edge of the target should be displaced forward by the same amount. However, if representational momentum occurs and is linked to anisotropies in the spatial representation of the target, then judgments of the final locations of the leading edge of the target and of the trailing edge of the target might be displaced by different amounts or even in different directions. Based on findings that a larger flash-lag effect occurs if the flashed object is aligned with the leading edge of the target than with the trailing edge of the target (Watanabe et al., 2001), it could be predicted that forward displacement should be larger for the leading edge of the target than for the trailing edge of the target.

\section{Method}

Participants Participants were undergraduates from the University of South Carolina, Upstate, who received partial course credit for participating. The research was approved by the Institutional Review Board at the University of South Carolina, and participants provided written informed consent prior to collection of their data. Participants were naïve to the purposes of the experiment until after their experimental trials were completed. Fifteen participants completed Experiment 1.
Apparatus The visual targets were displayed upon, and the data were collected with, a Gateway desktop computer connected to a 15-inch color monitor with a refresh rate of $60 \mathrm{~Hz}$ and a resolution of $1024 \times 768$ pixels. Participants' head and eye movements were not constrained, and the average viewing distance was approximately $60 \mathrm{~cm}$. Auditory cues were generated by the computer and presented over Sennheiser HD 280 Pro stereo headphones connected directly to the computer.

Stimuli The target was a square 20 pixels (approximately $0.83^{\circ}$ ) in width and in height and was presented on a white background. As shown in Fig. 1, there were five successive presentations of the target that implied consistent rightward motion or consistent leftward motion, and these are referred to as inducing stimuli. Each inducing stimulus was presented for $250 \mathrm{~ms}$, and there was a $250-\mathrm{ms}$ interstimulus interval between inducing stimuli (during which the display was blank); this resulted in an approximate target velocity of $3.32 \mathrm{deg} / \mathrm{s}$. Target motion could be rightward, leftward, downward, or upward. The vertical coordinates of rightwardinducing and leftward-inducing stimuli were approximately centered along the vertical axis of the display. For rightward motion, the first inducing stimulus appeared approximately midway between the left side and the center of the display, and the horizontal coordinates of each successive inducing stimulus were located 40 pixels (approximately $1.66^{\circ}$ ) to the right of the previous inducing stimulus; for leftward motion, the first inducing stimulus appeared approximately midway between the right side and the center of the display, and the horizontal coordinates of each successive inducing stimulus were located 40 pixels to the left of the previous inducing stimulus. The horizontal coordinates of downward-inducing

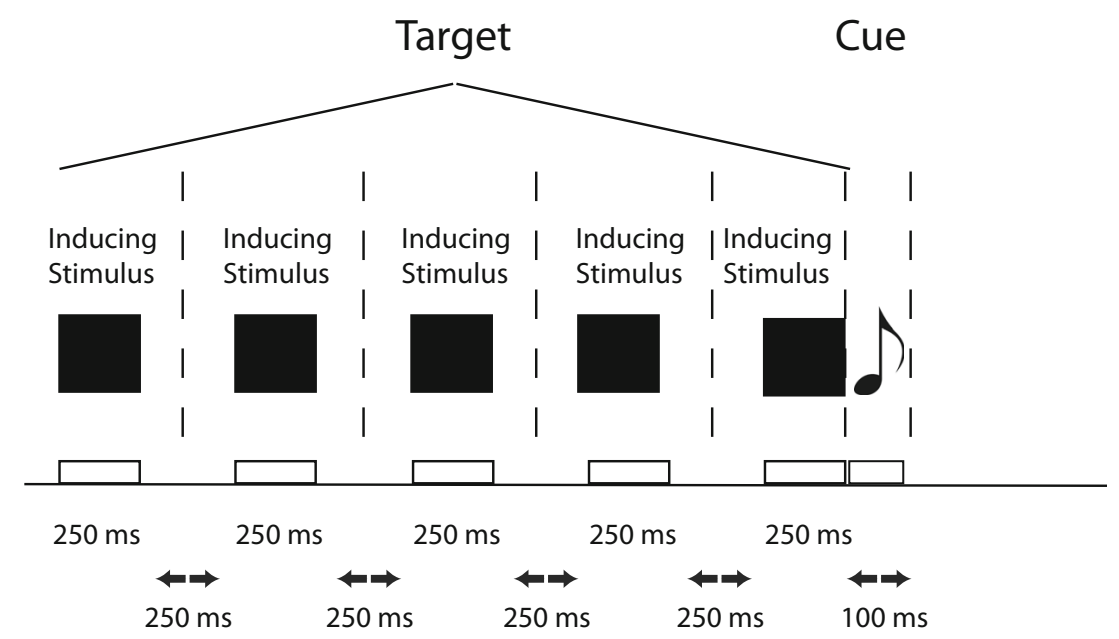

Fig. 1 Structure of a trial in Experiment 1. A sequence of five inducing stimuli, which composed the target, were presented. Each inducing stimulus was presented for $250 \mathrm{~ms}$, and there was $250 \mathrm{~ms}$ between successive inducing stimuli. Immediately after the final inducing

stimulus vanished, a cue consisting of an auditory tone was presented for $100 \mathrm{~ms}$. Participants then placed the cursor at the edge of the target indicated by the auditory cue 
and upward-inducing stimuli were approximately centered along the horizontal axis of the display. For downward motion, the first inducing stimulus appeared approximately one-third of the distance between the top and the center of the display, and the horizontal coordinates of each successive inducing stimulus were located 40 pixels below the previous inducing stimulus; for upward motion, the first inducing stimulus appeared approximately one-third of the distance between the bottom and the center of the display, and the vertical coordinates of each successive inducing stimulus were located 40 pixels (approximately $1.66^{\circ}$ ) above the previous inducing stimulus. The auditory cue was a $250-\mathrm{Hz}$ or $2000-\mathrm{Hz}$ tone that played for $100 \mathrm{~ms}$. Each participant received 80 trials ( 2 auditory cues $\times 4$ directions $\times 10$ replications) in a different random order.

Procedure Participants were first given a set of practice trials consisting of 10 trials randomly drawn from the experimental trials. Participants initiated each trial by pressing a designated key. There was a 1,000 ms pause, and then the target appeared and immediately began moving in a straight line across the display. Participants were instructed to watch the target, and a fixation point was not presented. Immediately after the target vanished, the auditory cue was presented. A high tone (2000 $\mathrm{Hz}$ ) instructed participants to position the cursor at the final location of the leading edge of the target (i.e., right edge of a rightward moving target, left edge of a leftward moving target, bottom edge of a downward moving target, top edge of an upward moving target), and a low tone $(250 \mathrm{~Hz})$ instructed participants to position the cursor at the final location of the trailing edge of the target (i.e., left edge of a rightward moving target, right edge of a leftward moving target, top edge of a downward moving target, bottom edge of an upward moving target). The cursor was positioned via movement of a computer mouse attached to the keyboard; the cursor was initially invisible but became visible after the target vanished. After positioning the cursor, participants clicked a button on the mouse to record the display coordinates of the cursor. Participants then initiated the next trial.

\section{Results}

Differences (in pixels) between the actual final location of the leading edge and the judged (indicated) final location of the leading edge of the target, and between the actual final location of the trailing edge and the judged (indicated) final location of the trailing edge of the target, were calculated along the axis of motion. Consistent with previous reports, these differences were referred to as $M$ displacement. Positively-signed $\mathrm{M}$ displacement indicated that the judged final position was beyond (i.e., located further in the direction of target motion) the actual final position (i.e., right of a rightward target, left of a leftward target, below a downward target, above an upward target), and negatively-signed $\mathrm{M}$ displacement indicated that the judged final position was behind (i.e., located in the direction opposite to target motion) the actual final position (i.e., left of a rightward target, right of a leftward target, above a downward target, below an upward target).

$\mathrm{M}$ displacements are shown in Figs. 2 and 3 and were analyzed in a 2 (edge: leading, trailing) $\times 4$ (direction: rightward, leftward, downward, upward) ANOVA, in which edge and direction were within-participant variables. $M$ displacement of the leading edge $(M=8.02, S E=3.66)$ was significantly less than $\mathrm{M}$ displacement of the trailing edge $(M=17.86, S E=2.78), F(1,14)=5.53, p<.035$, $M S E=525.02$, partial $\eta^{2}=0.28 . \mathrm{M}$ displacement was significantly larger than zero for the leading edge, $t(14)$ $=2.20, p<.046$, and significantly larger than zero for the trailing edge, $t(14)=6.43, p<.001$.

$\mathrm{M}$ displacement was influenced by direction, $F(3,42)=$ 8.16, $p<.001, M S E=86.38$, partial $\eta^{2}=0.37$, and simple pairwise comparisons $(p<.05)$ suggested $M$ displacement of upward $(M=6.07, S E=3.47)$ motion was less than $M$ displacement of rightward $(M=15.71, S E=2.18)$, leftward $(M=$ 16.89, $S E=3.26)$, or downward $(M=13.09, S E=2.42)$ motion, and that $\mathrm{M}$ displacement of downward motion was less than $M$ displacement of leftward motion. Edge $\times$ Direction was not significant, $F(3,42)=2.21, p>.10$.

\section{Discussion}

Judgments of the final locations of the leading edge of the target and the trailing edge of the target were displaced forward. However, the magnitude of displacement was larger for judgments of the trailing edge, and so effects of representational momentum were not equal across all parts of the target. The larger forward displacement of the trailing edge does not initially seem consistent with the larger flash-lag effect for flashed objects aligned with the leading edge of a moving target reported by Watanabe et al. (2001), but the reason for this is not yet clear; a possible explanation will be suggested in

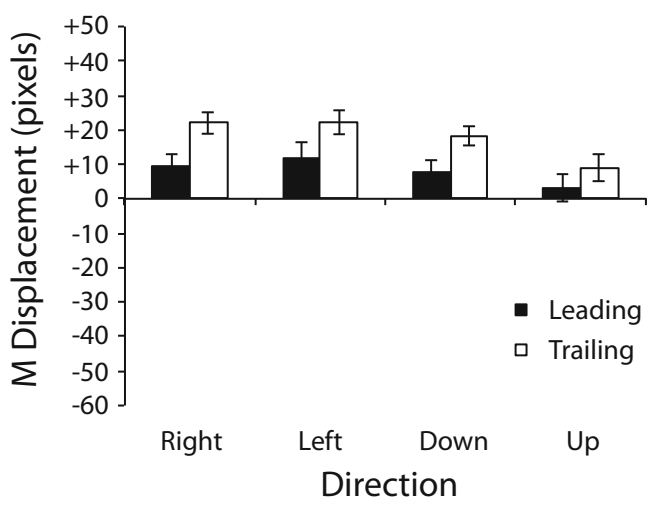

Fig. $2 \mathrm{M}$ displacement as a function of direction in Experiment 1. M displacement for the leading edge of the target is indicated with black columns, and $\mathrm{M}$ displacement for the trailing edge of the target is indicated with white columns. Error bars reflect standard error of the mean 
A

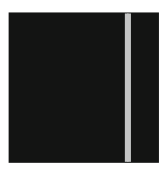

B

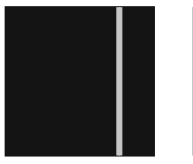

C

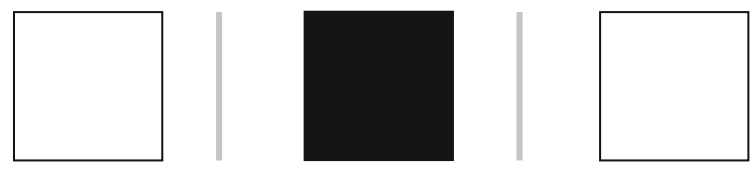

D

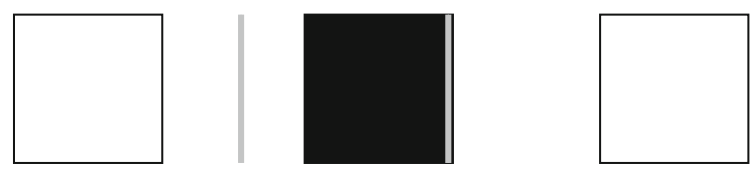

Trailing Edge

Leading Edge

Target Motion (Rightward)

Fig. 3 Average displacements of leading edges and trailing edges in Experiments 1, 2, 3, and 4. Panels A, B, C, and $\mathbf{D}$ show the results from Experiments 1, 2, 3, and 4, respectively. Average displacements are collapsed across direction and shown using a rightward target. Black squares indicate final location of the target, and left and right vertical gray bars indicate the average judged locations of the trailing edge and leading edge, respectively. In panels $\mathbf{C}$ and $\mathbf{D}$, white squares indicate the locations of the stationary objects relative to the final target location

the General Discussion. Interestingly, the larger forward displacement of the trailing edge than of the leading edge is consistent with a hypothesis that the target is remembered as being slightly smaller than its actual size. This is consistent with a compression of size along the axis of motion for figures in anorthoscopic perception (i.e., moving figures viewed through a slit in which only a portion of the target is visually available at any given time; Anstis \& Atkinson, 1967; for review, see Rock, 1997) and with findings from memory psychophysics in which the exponent relating remembered area to physical area is smaller than the exponent relating perceived area to physical area (for reviews, see Algom, 1992; Hubbard, 1994). The effect of direction is consistent with that reported in previous studies (e.g., Hubbard, 1990; Hubbard \& Bharucha, 1988); namely, forward displacement is larger for horizontal motion than for vertical motion, and within vertical motion, forward displacement is larger for downward motion than for upward motion.

\section{Experiment 2}

Whether participants judged the final location of the leading edge or the final location of the trailing edge of the target was a within-participants variable in Experiment 1. Participants did not know until after the target vanished whether they would indicate the final location of the leading edge or the trailing edge, and so attention to the target was presumably distributed over both the leading edge and the trailing edge (or focused between the leading and trailing edges at the center) of the target. However, it is possible that a different pattern or magnitude of displacement might occur if participants allocated attention to just a single edge of the target. Thus, it would be useful to replicate the finding of a larger forward displacement for the trailing edge than for the forward edge when edge is a between-participants variable. Accordingly, in Experiment 2, one group of participants always judged the final location of the leading edge of the target, and another group of participants always judged the final location of the trailing edge of the target. Although the auditory cue used in Experiment 1 was no longer necessary for signaling whether the location of the leading edge or the trailing edge should be indicated, it was retained within each trial in order to make trial structure in Experiment 2 as similar as possible to trial structure in Experiment 1 (and so participants in the leading edge condition always heard a high tone when the target vanished, and participants in the trailing edge condition always heard a low tone when the target vanished).

\section{Method}

Participants Participants were 30 undergraduates from the same participant pool used in Experiment 1, and none had participated in Experiment 1. Fifteen participants were in the leading edge condition, and 15 participants were in the trailing edge condition.

Apparatus The apparatus was the same as in Experiment 1.

Stimuli The stimuli were the same as in Experiment 1, with the following exceptions: Each participant judged only the final location of the leading edge of the target or judged only the final location of the trailing edge of the target. Each participant received 40 trials ( 4 directions $\times 10$ replications) in a different random order.

Procedure The procedure was the same as in Experiment 1, with the following exceptions: Participants in the leading edge condition always indicated the final location of the leading 
edge of the target and heard a high auditory tone when the target vanished, and participants in the trailing edge condition always indicated the final location of the trailing edge of the target and heard a low auditory tone when the target vanished.

\section{Results}

M displacements were calculated as in Experiment 1. M displacements are shown in Figs. 3 and 4 and were analyzed in a 2 (edge: leading, trailing) $\times 4$ (direction: rightward, leftward, downward, upward) ANOVA, in which edge was a betweenparticipants variable and direction was a within-participants variable. $M$ displacement of the leading edge $(M=5.54, S E$ = 4.16) was significantly less than $\mathrm{M}$ displacement of the trailing edge $(M=17.73, S E=4.16), F(1,28)=4.29, p<$ $.05, M S E=259.46$, partial $\eta^{2}=0.13$. M displacement was significantly larger than zero for the leading edge, $t(14)=2.72$, $p<.016$, and significantly larger than zero for the trailing edge, $t(14)=3.21, p<.006$.

$\mathrm{M}$ displacement was influenced by direction, $F(3,26)=$ $6.75, p<.002, M S E=86.38$, partial $\eta^{2}=0.44$, and simple pairwise comparisons $(p<.05)$ suggested $\mathrm{M}$ displacement of upward $(M=7.58, S E=2.49)$ motion was less than $M$ displacement of rightward $(M=13.30, S E=3.47)$, leftward $(M=$ $14.54, S E=3.38)$, or downward $(M=11.12, S E=2.81)$ motion, and that $\mathrm{M}$ displacement of downward motion was less than $M$ displacement of leftward motion. Edge $\times$ Direction was not significant, $F(3,42)=2.21, p>.10$.

\section{Discussion}

As in Experiment 1, judgments of the final locations of the leading edge and trailing edge of the target were displaced forward, and forward displacement of the leading edge was smaller than forward displacement of the trailing edge. As in Experiment 1, M displacement was larger for horizontal

\footnotetext{
${ }^{2}$ Although the displacement pattern in Experiment 1 is consistent with attentional momentum, the results of Experiment 2 rule out an explanation that is based on attentional momentum. In Experiment 1, participants did not know before the target vanished whether they would indicate the final location of the leading edge or the final location of the trailing edge. Thus, an optimal strategy would have involved attending near the center of the target. With this strategy, the leading edge was located farther in the direction of motion (i.e., compatible with the current direction of target motion), whereas the trailing edge was located in the direction opposite to motion (i.e., incompatible with the current direction of target motion). Given that detection is facilitated when a stimulus is located along the current path of motion and not facilitated when a stimulus is located in a different direction, detection of the final location of the leading edge could have been facilitated (perhaps allowing less motion extrapolation before judgment), and detection of the final location of trialing edge would not have been facilitated (perhaps allowing more motion extrapolation before judgment). However, in Experiment 2, participants knew before the target appeared which edge they would have to indicate, and so rather than focusing on the center of the target, they presumably focused on the edge they would have to indicate, thus removing the need to shift attention forward or backward to the queried edge.
}

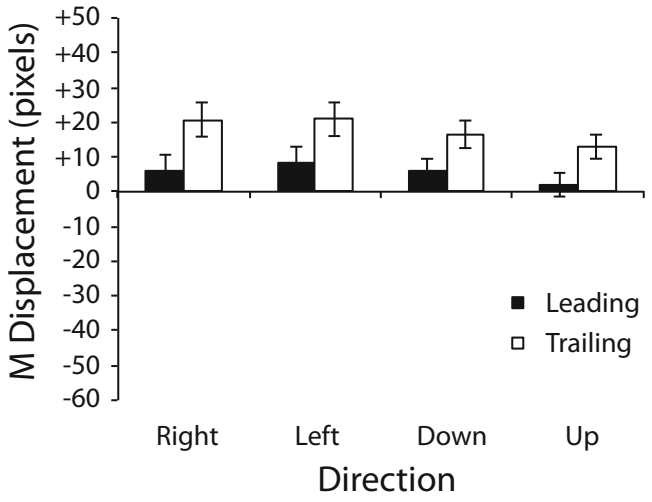

Fig. $4 \mathrm{M}$ displacement as a function of direction in Experiment 2. $\mathrm{M}$ displacement for the leading edge of the target is indicated with black columns, and $\mathrm{M}$ displacement for the trailing edge of the target is indicated with white columns. Error bars reflect standard error of the mean

motion than for vertical motion, and for vertical motion, larger for downward motion than for upward motion. Comparison of Figs. 2 and 4 shows that forward displacement of the leading edge and trailing edge in Experiment 2 were very similar to forward displacement of the leading edge and trailing edge in Experiment 1, and this suggests that whether participants attended to a single edge or to multiple edges (or perhaps to the center) of the target did not influence displacement. The similarities in the magnitudes of displacement across Experiments 1 and 2 are consistent with previous experiments in which the type of judgment (e.g., initial location or final location of the target) was treated as a within-participants variable and as a between-participants variable (Hubbard \& Motes, 2002). The results of Experiments 1 and 2 suggest that representational momentum is linked to anisotropy in spatial representation across the surface of the target. ${ }^{2}$ The forward displacement of the leading edge (and indeed, the existence of representational momentum more generally) suggests this anisotropy might extend into space beyond the edges of the target, and this possibility is examined in Experiment 3.

\section{Experiment 3}

The extent to which the anisotropy observed in Experiments 1 and 2 might extend along the (previous or potential) path of target motion beyond the edges of the target is not clear. Previous findings in the flash-lag effect (Watanabe et al., 2001) and representational momentum (Hubbard, 2008) literature suggest that anisotropy of visual space related to target motion can influence nontarget stimuli that are close but not immediately adjacent to the target. In Experiment 3, the same targets used in Experiments 1 and 2 were presented, and in addition, two stationary objects along the path of motion were also presented on each trial. The stationary objects were the same shape and size as, but a different color than, the target; one 
was located in front of the target and the other was located an equal distance behind the target. The stationary objects appeared when the final inducing stimulus appeared and vanished when the final inducing stimulus vanished. As in Experiment 1, an auditory cue was presented when the target vanished and instructed participants which location to indicate. If the auditory cue was a high tone, participants indicated the location of the nearest edge of the stationary object in front of the target, and if the auditory cue was a low tone, participants indicated the location of the nearest edge of the stationary object behind the target. If the pattern observed in Experiments 1 and 2 extends beyond the edges of the target, then forward displacement of the object in front of the target should be smaller than forward displacement of the object behind the target.

\section{Method}

Participants The participants were 15 undergraduates from the same participant pool used in Experiment 1, and none had participated in Experiments 1 or 2.

Apparatus The apparatus was the same as in Experiment 1.

Stimuli The moving targets and auditory cues were the same as in Experiment 1. Additionally, two stationary objects were presented on each trial: one in a leading position in front of the target (the leading edge condition), and one in a trailing position behind the target (the trailing edge condition). Each stationary object was a square 20 pixels (approximately $0.83^{\circ}$ ) in width and height (the same as the target); the interior of the square was white, and the edges of the square were 1-pixel thick black lines. The stationary objects were only visible during the presentation of the final inducing stimulus in order to approximate the brief presentations of objects in Watanabe et al. (2001), Whitney and Cavanagh (2000), and Hubbard (2008); additionally, it was hoped that a briefer presentation would decrease the likelihood of a stationary object being encoded as a stable landmark or reference point and increase the likelihood that the representation of a stationary object would be influenced by target motion. The distance between the closest edge of the target and the closest edge of each object was 20 pixels (i.e., the leading edge of the target was 20 pixels [approximately $0.83^{\circ}$ ] from the nearest edge of stationary object in front of the target, and the trailing edge of the target was 20 pixels [approximately $0.83^{\circ}$ ] from the nearest edge of the stationary object behind the target). Each participant received 80 trials $(2$ auditory cues $\times 4$ directions $\times 10$ replications) in a different random order.

Procedure The procedure was the same as in Experiment 1, with the following exceptions: Stationary objects the same size and shape as the target appeared in front of and behind the target at the same time that the final inducing stimulus appeared and vanished at the same time the final inducing stimulus vanished. If a high tone $(2000 \mathrm{~Hz})$ was presented, participants positioned the cursor at the location of the nearest edge of the stationary object that was in front of the target (the leading edge condition), and if a low tone $(250 \mathrm{~Hz})$ was presented, participants positioned the cursor at the location of the nearest edge of the stationary object that was behind the target (the trailing edge condition).

\section{Results}

M displacements were calculated as in Experiment 1, with the following exceptions: The "leading edge" was the nearest edge of the stationary object in front of the target, and the "trailing edge" was the nearest edge of the stationary object behind the target. M displacements are shown in Figs. 3 and 5 and were analyzed in a 2 (edge: leading, trailing) $\times 4$ (direction: rightward, leftward, downward, upward) ANOVA, in which edge and direction were within-participant variables. $\mathrm{M}$ displacement of the leading edge $(M=-12.61, S E=$ 3.12) was significantly less than $M$ displacement of the trailing edge $(M=7.41, S E=3.15), F(1,14)=11.61, p<$ $.004, M S E=1035.97$, partial $\eta^{2}=0.45$. M displacement was significantly smaller than zero for the leading edge, $t(14)=$ $-4.04, p<.001$, and significantly larger than zero for the trailing edge, $t(14)=2.35, p<.034$.

$\mathrm{M}$ displacement was influenced by direction, $F(3,42)=$ 8.58, $p<.001$, MSE $=64.18$, partial $\eta^{2}=0.38$, and simple pairwise comparisons $(p<.05)$ suggested $\mathrm{M}$ displacement of upward $(M=-7.49, S E=1.16)$ motion was different from $\mathrm{M}$ displacement of rightward $(M=-3.55, S E=1.40)$, leftward $(M=-2.27, S E=1.73)$, or downward $(M=2.89, S E=1.94)$ motion, and that $\mathrm{M}$ displacement of downward motion was different from $\mathrm{M}$ displacement of leftward or rightward motion. Edge $\times$ Direction was not significant, $F(3$, 42) $=1.37, p>.26$.

\section{Discussion}

Displacement of the judged location of the nearest edge of the object in front of the target was backward (i.e., in the direction opposite to target motion), and displacement of the judged location of the nearest edge of the object behind the target was forward (i.e., in the direction of target motion); both of these displacements were significantly different from zero. However, both of these displacements are toward the target, and so an alternative hypothesis is that these displacements reflect a bias toward the target (e.g., a landmark attraction effect) and are not related to representational momentum or target motion per se. If this alternative hypothesis is correct, then the magnitude of displacement should not be influenced by whether the object was in front of or behind the target. In order to test this hypothesis, displacements for objects in front 


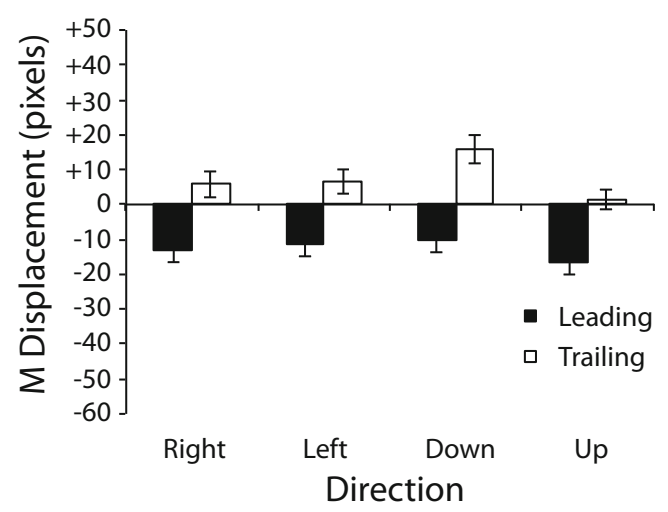

Fig. $5 \mathrm{M}$ displacement as a function of direction in Experiment 3. M displacement for the nearest edge of the object in front of (leading) the target is indicated with black columns, and $\mathrm{M}$ displacement for the nearest edge of the object behind (trailing) the target is indicated with white columns. Error bars reflect standard error of the mean

of the target were multiplied by -1 (so that displacements toward the target would have a positive sign for both leading edge and trailing edge conditions) and compared to displacements for objects behind the target. Judgments of the leading edge exhibited a larger magnitude of displacement than did judgments of the trailing edge, $t(14)=-2.36, p<.033$, and so the alternative hypothesis that displacements reflected a general bias toward the target and were not related to the direction of target motion can be rejected. Also, the negative displacement for the edge of an object in front of the target is consistent with underestimation of time-to-contact (e.g., McLeod \& Ross, 1983; Schiff \& Detwiler 1979), that is, a compression of visual space between the target and the object.

Displacement in judged location for downward motion was more forward than was displacement in judged location for other directions; indeed, downward motion was the only direction to have an average displacement that was positive. It is possible that downward motion in Experiment 3 might have resulted in larger positive displacement than did motion in other directions because of a combination of the effects of representational momentum, gravitational attraction, and landmark attraction. The judged location of a nearby stationary object can be shifted in the direction of representational momentum of a moving target (Hubbard, 2008), and, similarly, the judged location of a target is shifted in the direction of a landmark (Hubbard \& Ruppel, 1999) and in the direction of implied gravitational attraction (Hubbard, 1997). In downward motion in Experiment 3, these three influences operate in the same direction (downward), and so they sum and the resulting displacement is relatively more forward. Influences of representational momentum and the direction of implied gravitational attraction presumably also summed with downward motion in Experiments 1 and 2, but the absence of a potential landmark effect in Experiments 1 and 2 resulted in a relatively smaller forward displacement. The magnitude of displacement was larger in Experiment 3 than in Experiments
1 and 2, and this suggests anisotropy might be larger at a greater distance from the target, and this possibility is examined in Experiment 4.

\section{Experiment 4}

The magnitudes of displacement in Experiment 3 were larger than the magnitudes of displacement in Experiments 1 and 2. It is possible this occurred because the locations to be indicated were beyond the edges of the target in Experiment 3 but were part of the target in Experiments 1 and 2. In such a view, there might be an effect of distance from the target on anisotropy of nearby visual space, with distances farther away from the target exhibiting larger anisotropy. Accordingly, Experiment 4 was the same as Experiment 3, except that the high and low auditory cues instructed participants to indicate the farthest, rather than the nearest, edges of the object in front of or behind, respectively, the target. The farthest edges of the stationary objects were twice the distance of the nearest edges of the stationary object from the target, and so if anisotropy in nearby visual space increases as distance from the target increases, then displacements in Experiment 4 should be larger in magnitude than displacements in Experiment 3. Alternatively, if anisotropy in nearby visual space is not influenced by the distance between the target and the location to be indicated, and the larger magnitudes of displacement in Experiment 3 than in Experiments 1 and 2 only reflected whether or not the judged location was on or separate from the target, then displacements in Experiment 4 should not differ from displacements in Experiment 3.

\section{Method}

Participants Participants were 16 undergraduates from the same participant pool used in Experiment 1, and none had participated in Experiments 1, 2, or 3.

Apparatus The apparatus was the same as in Experiment 1.

Stimuli The moving targets, stationary objects, and auditory cues were the same as in Experiment 3. Each participant received 80 trials $(2$ auditory cues $\times 4$ directions $\times 10$ replications) in a different random order.

Procedure The procedure was the same as in Experiment 3, with the following exceptions: If a high tone $(2000 \mathrm{~Hz})$ was presented, participants positioned the cursor at the location of the farthest edge of the stationary object that was in front of the target (the leading edge condition), and if a low tone $(250 \mathrm{~Hz})$ was presented, participants positioned the cursor at the location of the farthest edge of the stationary object that was behind the target (the trailing edge condition). 


\section{Results}

M displacements were calculated as in Experiment 1, with the following exceptions: The "leading edge" was the farthest edge of the stationary object in front of the target, and the "trailing edge" was the farthest edge of the stationary object behind the target. $\mathrm{M}$ displacements are shown in Figs. 3 and 6 and were analyzed in a 2 (edge: leading, trailing) $\times 4$ (direction: rightward, leftward, downward, upward) ANOVA, in which edge and direction were within-participant variables. $\mathrm{M}$ displacement of the leading edge $(M=-44.83, S E=$ 4.94) was significantly less than $M$ displacement of the trailing edge $(M=32.62, S E=4.81), F(1,15)=92.13, p<$ $.0001, M S E=2079.05$, partial $\eta^{2}=0.86 . \mathrm{M}$ displacement was significantly smaller than zero for the leading edge, $t(14)=$ $-9.08, p<.001$, and significantly larger than zero for the trailing edge, $t(14)=6.78, p<.001$.

$\mathrm{M}$ displacement was influenced by direction, $F(3,45)=$ 5.54, $p<.003, M S E=159.02$, partial $\eta^{2}=0.27$, and simple pairwise comparisons $(p<.05)$ suggested $\mathrm{M}$ displacement of upward $(M=-12.79, S E=3.33)$ motion was different from $\mathrm{M}$ displacement of rightward $(M=-4.64, S E=3.82)$, leftward $(M=-6.82, S E=3.50)$, or downward $(M=-0.17, S E=2.62)$ motion, and that $\mathrm{M}$ displacement of rightward motion was different from $\mathrm{M}$ displacement of downward motion. Edge $\times$ Direction was not significant, $F(3,45)=0.65, p>.10$.

\section{Discussion}

As in Experiment 3, displacement of the judged location of the farthest edge of the object in front of the target was backward, and displacement of the judged location of the farthest edge of the object behind the target was forward; both of these displacements were significantly different from zero. As in Experiment 3, the displacements for objects in front of the

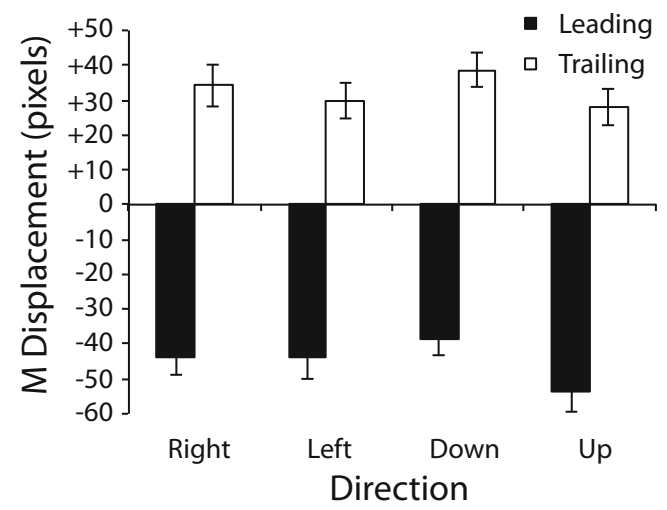

Fig. $6 \mathrm{M}$ displacement as a function of direction in Experiment 4. M displacement for the farthest edge of the object in front of (leading) the target is indicated with black columns, and $\mathrm{M}$ displacement for the farthest edge of the object behind (trailing) the target is indicated with white columns. Error bars reflect standard error of the mean target were multiplied by -1 (so that displacements toward the target would have a positive sign for both leading edge and trailing edge conditions) and compared to displacements for objects behind the target. The leading edge exhibited a larger magnitude of displacement than did the trailing edge, $t(15)=2.30, p<.05$; this is consistent with Experiment 3 and suggests that displacement of the object does not just reflect a general bias toward the location of the target but is specifically related to the direction of motion of the target. Although displacement for downward targets was negative in Experiment 4 , this displacement was relatively more forward than was displacement for other directions, and this is consistent with findings in Experiment 3, and the suggestion that with downward motion the effects of representational momentum, implied gravitational attraction, and landmark attraction all operated in the same direction and summed to produce a relatively more forward (less backward) displacement.

The magnitude of backward displacement for objects in front of the target, and the magnitude of forward displacement for objects behind the target, were larger in Experiment 4 than in Experiment 3. This pattern is consistent with the notion that anisotropy in visual space increases as distance from the target increases. Of course, such an increase would not be expected to continue indefinitely with continued increases in distance from the target but would be expected to asymptote, plateau, or decrease once some threshold distance was reached. Determination of this threshold, and of variables that influence such a threshold, remains a topic for future research. Even so, the displacement was larger than that typically observed in studies of representational momentum (although see Fig. 3 in Hubbard \& Bharucha, 1988), and displacements of the objects spanned most of the distance between those objects and the target. Given that visual acuity decreases as the distance from the fovea increases, one possible hypothesis is that larger displacement with increased distance merely reflects decreased acuity in peripheral areas of the retina. However, it is not clear how such a hypothesis would account for differences between the near edges in Experiment 3 and between the far edges in Experiment 4, as all objects were the same size and the same distance from the target. Regardless of the mechanism, though, anisotropy related to motion of the target can influence spatial representation of nontarget stimuli in nearby visual space.

\section{General discussion}

When displacements of the final locations of the leading edge and the trailing edge of a single rigid target are measured, judgments of the final locations of the leading edge and the trailing edge are displaced forward, but displacement of the leading edge is smaller than displacement of the trailing edge (Experiments 1 and 2). When displacements of the locations 
of the nearest edge and the farthest edge of a stationary object in front of or behind the final location of the target are measured, judgments of the edges of an object in front of the target are displaced backward and judgments of the edges of an object behind the target are displaced forward; furthermore, the magnitude of displacement is larger if the edges are farther from the target (Experiments 3 and 4). Although the edges of an object in front of or behind the final location of the target are displaced toward the target (i.e., backward for an object in front of the target, forward for an object behind the target), the hypothesis that these displacements reflect just a general bias toward the target location and are not influenced by target motion or representational momentum can be rejected, as such a hypothesis predicts there should be no difference between displacements, but in Experiments 3 and 4, the magnitude of displacement for an object in front of the target was larger than the magnitude of displacement for an object behind the target. It appears that a moving target (or representational momentum for that target) is linked with anisotropies in nearby visual space.

In Experiments 1 and 2, the magnitudes of displacement of the leading edge of the target were smaller than the magnitudes of displacement of the trailing edge of the target, whereas in Experiments 3 and 4, the magnitudes of displacement of the edges of objects in front of the target were larger than the magnitudes of displacement of the edges of objects behind the target. Such results might initially appear inconsistent, but closer examination reveals an unexpected consistency: In all experiments, the trailing edge (of the target or the object behind the target) was displaced further forward than was the leading edge (of the target or the object in front of the target). The forward displacement of the leading edge of the target (Experiments 1 and 2), and the backward displacement of an edge of an object in front of the target (Experiments 3 and 4), suggest that the space between the target and the object in front of the target is contracted or compressed; furthermore, this contraction or compression increases with distance from the target. As noted earlier, such a contraction or compression is consistent with findings of an underestimation of time-to-contact. As also noted earlier, the smaller magnitude of displacement for the leading edge of target, coupled with the larger magnitude of displacement for the trailing edge of the target, is consistent with findings that (a) perceived size of an object is decreased (compressed) along the axis of motion in anorthoscopic perception and (b) remembered size of an object is smaller than the physical or perceived size in memory psychophysics.

It is tempting to conclude that the smaller forward displacement for the leading edge than for the trailing edge of targets in Experiments 1 and 2 suggests that representation of target shape and size is distorted. Similarly, it is tempting to conclude that smaller displacement for the near edge than for the far edge of objects in Experiments 3 and 4 suggests that representation of object shape and size is distorted. However, participants indicated the location of only one edge on any given trial, and so the current data do not unequivocally support a distortion of shape or size. Indeed, a consideration of Watanabe and Yokoi (2006) suggests that target shape and size and objects shape and size might not be distorted; rather, localization of (the undistorted shape of) the target or object might be distorted. In this view, representational momentum of the target decreases perceived distance between the target and the object in front of the target, and this is represented as a veridical target position and a decreased distance to the object (i.e., negative displacement of the object). As noted earlier, such a decrease in represented distance is consistent with studies finding a presaccadic compression of space, and so representational momentum of the target might contribute to a remapping of the space encompassing the target and the nearby space that the target is expected to enter (cf. Matsumiya \& Uchikawa, 2003). Of course, whether such a remapping involves changes to objects or changes to visual space is not entirely clear.

The smaller forward displacement for the leading edge of the target than for the trailing edge of the target observed in Experiments 1 and 2 initially seems inconsistent with the larger flash-lag effect reported when the flashed object was aligned with the leading edge of the moving target than when the flashed object was aligned with the trailing edge of the moving target in Watanabe et al. (2001; Watanabe, 2004). A flashed object aligned with the leading edge of a moving target is still perceived as aligned with some part of the moving target (e.g., in Watanabe et al., 2001, the flashed object aligned with the leading edge of a square was perceived to lag by 0.42 degrees, but the length of the square moving target was 1.92 degrees, and so a flashed object aligned with the leading edge could still be perceived as aligned with the front half of the target (i.e., forward of the horizontal center of the horizontally moving target), whereas a flashed object aligned with the trailing edge of the target was not perceived as aligned with any part of the moving target. One possibility is that there was a center bias such that the flashed object was biased toward the central vertical axis of the horizontally moving target (cf. Experiment 5 in Hubbard \& Ruppel, 2000). For targets at the leading edge, the flash-lag effect and center bias operated in the same direction (increasing the magnitude of the flashlag effect), and for targets at the trailing edge, the flash-lag effect and center bias operated in opposite directions (decreasing the magnitude of the flash-lag effect).

The differences in magnitudes of displacement for the leading edge of the target and the trailing edge of the target, and for edges of objects in front of the target and objects behind the target, suggest that displacement is related to the direction of motion of the target and does not reflect just a general bias toward the location of the target. Furthermore, magnitudes of displacement are relatively smaller for the edges of the target and increase with increases in the distance of the judged location from the moving target. However, it is not clear whether 
the observed anisotropies are a cause or an effect of representational momentum. It is possible that representational momentum results in a distortion in memory for a target that leads to anisotropies in spatial representation; alternatively, it is possible that representational momentum reflects a broader anisotropy or distortion of spatial representation (e.g., representational momentum might result from a general contraction of space in front of the target and/or an expansion of space behind the target). Also, it is not clear how the current findings regarding representational momentum in visual space might be related to potential anisotropies in nonvisual (e.g., auditory, tactile) space. Regardless, representational momentum is linked with anisotropies along the axis of motion in visual space, and different parts of the target and different parts of nearby space appear to exhibit different magnitudes of displacement.

Previous studies of representational momentum for visual location generally relied on judgments of the center of the target or judgments of a subsequent probe that was the same shape and size as the target, and few studies considered if representational momentum of a moving target might affect nontarget stimuli. The experiments reported here involved judgments of the location of the leading edge or trailing edge of a target or judgments of the location of the edge of a stationary object in front of or behind the target. A gradient of representational momentum was found, such that forward displacement decreased (and reversed) as the location of the queried edge moved from behind to in front of the target. Displacement extended beyond the target, as edges of objects in front of the target were displaced backward and edges of objects behind the target were displaced forward; however, these latter displacements were not just due to a general bias toward the target but depended upon the direction of target motion, as the magnitude of backward displacement of objects in front of the target was larger than the magnitude of forward displacement of objects behind the target. These displacements are consistent with previous findings regarding (a) underestimation of time-to-contact, (b) smaller perceived target sizes in anorthoscopic perception, and (c) smaller remembered target sizes in memory psychophysics. Whether representational momentum leads to anisotropy of perceived space, or whether anisotropy of perceived space leads to representational momentum, is not yet clear. Regardless, representational momentum for a moving target is linked to anisotropies in the perceived space of and surrounding that target.

\section{References}

Algom, D. (1992). Memory psychophysics: An examination of its perceptual and cognitive prospects. In D. Algom (Ed.), Psychophysical approaches to cognition (pp. 441-513). Amsterdam, Netherlands: Elsevier.
Anstis, S. M., \& Atkinson, J. (1967). Distortions in moving figures viewed through a stationary slit. The American Journal of Psychology, 80(4), 572-585.

Aznar-Casanova, J. A., \& Bernardino, L. G. (in press). Perceptual anisotropies in visual space. In T. L. Hubbard (Ed.), Spatial biases in perception and cognition. New York, NY: Cambridge University Press.

Ball, K., \& Sekuler, R. (1980). Models of stimulus uncertainty in motion perception. Psychological Review, 87(5), 435-469. https://doi.org/ 10.1037/0033-295X.87.5.435

Freyd, J. J., \& Johnson, J. Q. (1987). Probing the time course of representational momentum. Journal of Experimental Psychology: Learning, Memory, and Cognition, 13, 259-268. https://doi.org/ 10.1037/0278-7393.13.2.259

Gray, R., \& Thornton, I. M. (2001). Exploring the link between time to collision and representational momentum. Perception, 30, 10071022. https://doi.org/10.1068/p3220

Gros, B. L., Blake, R., \& Hiris, E. (1998). Anisotropies in visual motion perception: A fresh look. Journal of the Optical Society of America A, 15(8), 2003-2011. https://doi.org/10.1364/JOSAA.15.002003

Hubbard, T. L. (1990). Cognitive representation of linear motion: Possible direction and gravity effects in judged displacement. Memory \& Cognition, 18, 299-309. https://doi.org/10.3758/ BF03213883

Hubbard, T. L. (1993). The effects of context on visual representational momentum. Memory \& Cognition, 21, 103-114. https://doi.org/10. 3758/BF03211169

Hubbard, T. L. (1994). Memory psychophysics. Psychological Research, 56(4), 237-250. https://doi.org/10.1007/BF00419654

Hubbard, T. L. (1995). Environmental invariants in the representation of motion: Implied dynamics and representational momentum, gravity, friction, and centripetal force. Psychonomic Bulletin \& Review, 2, $322-338$.

Hubbard, T. L. (1997). Target size and displacement along the axis of implied gravitational attraction: Effects of implied weight and evidence of representational gravity. Journal of Experimental Psychology: Learning, Memory, and Cognition, 23, 1484-1493. https://doi.org/10.1037/0278-7393.23.6.1484

Hubbard, T. L. (2005). Representational momentum and related displacements in spatial memory: A review of the findings. Psychonomic Bulletin \& Review, 12, 822-851. https://doi.org/10.3758/ BF03196775

Hubbard, T. L. (2008). Representational momentum contributes to motion induced mislocalization of stationary objects. Visual Cognition, 16, 44-67. https://doi.org/10.1080/13506280601155468

Hubbard, T. L. (2014a). Forms of momentum across space: Representational, operational, and attentional. Psychonomic Bulletin \& Review, 21, 1371-1403. https://doi.org/10.3758/ s13423-014-0624-3

Hubbard, T. L. (2014b). The flash-lag effect and related mislocalizations: Findings, properties, and theories. Psychological Bulletin, 140, 308 338. https://doi.org/10.1037/a0032899

Hubbard, T. L., \& Bharucha, J. J. (1988). Judged displacement in apparent vertical and horizontal motion. Perception \& Psychophysics, 44, 211-221. https://doi.org/10.3758/BF03206290

Hubbard, T. L., \& Motes, M. A. (2002). Does representational momentum reflect a distortion of the length or the endpoint of a trajectory? Cognition, 82, B89-B99. https://doi.org/10.1016/S0010-0277(01) 00156-1

Hubbard, T. L., \& Ruppel, S. E. (1999). Representational momentum and the landmark attraction effect. Canadian Journal of Experimental Psychology, 53, 242-256. https://doi.org/10.1037/h0087313

Hubbard, T. L., \& Ruppel, S. E. (2000). Spatial memory averaging, the landmark attraction effect, and representational gravity. Psychological Research/ Psychologische Forschung, 64, 41-55. https://doi.org/10.1007/s004260000029 
Jancke, D., \& Erlhagen, W. (2010). Bridging the gap: A model of common neural mechanisms underlying the Fröhlich effect, the flash-lag effect, and the representational momentum effect. In R. Nijhawan \& B. Khurana (Eds.), Space and time in perception and action (pp. 422-440). Cambridge, UK: Cambridge University Press.

Kerzel, D. (2000). Eye movements and visible persistence explain the mislocalization of the final position of a moving target. Vision Research, 40, 3703-3715. https://doi.org/10.1016/S0042-6989(00) 00226-1

Kerzel, D., Jordan, J. S., \& Müsseler, J. (2001). The role of perception in the mislocalization of the final position of a moving target. Journal of Experimental Psychology: Human Perception and Performance, 27(4), 829-840. https://doi.org/10.1037/0096-1523.27.4.829

Lappe, M., Awater, H., \& Krekelberg, B. (2000). Postsaccadic visual references generate presaccadic compression of space. Nature, 403(6772), 892-895. https://doi.org/10.1038/35002588

Matsumiya, K., \& Uchikawa, K. (2003). The role of presaccadic compression of visual space in spatial remapping across saccadic eye movements. Vision Research, 43(18), 1969-1981. https://doi.org/ 10.1016/S0042-6989(03)00301-8

McLeod, R. W., \& Ross, H. E. (1983). Optic-flow and cognitive factors in time-to-collision estimates. Perception, 12(4), 417-423. https://doi. org $/ 10.1068 / p 120417$

Müsseler, J., Stork, S., \& Kerzel, D. (2002). Comparing mislocalizations with moving stimuli: The Fröhlich effect, the flash-lag, and representational momentum. Visual Cognition, 9, 120-138. https://doi. org/10.1080/13506280143000359

Posner, M. I., \& Cohen, Y. (1984). Components of visual orienting. In H. Bouma \& D. G. Bouwhuis (Eds.), Attention and performance X: Control of language processes (pp. 531-556). Hove, UK: Erlbaum.

Posner, M. I., Rafal, R. D., Choate, L., \& Vaughan, J. (1985). Inhibition of return: Neural basis and function. Cognitive Neuropsychology, 2, 211-228. https://doi.org/10.1080/02643298508252866
Pratt, J., Spalek, T. M., \& Bradshaw, F. (1999). The time to detect targets at inhibited and noninhibited locations: Preliminary evidence for attentional momentum. Journal of Experimental Psychology: Human Perception and Performance, 25(3), 730-746. https://doi. org/10.1037/0096-1523.25.3.730

Rock, I. (1997). Anorthoscopic perception. In I. Rock (Ed.), Indirect perception (pp. 107-124) Cambridge, MA: MIT Press.

Ross, J., Morrone, M. C., \& Burr, D. C. (1997). Compression of visual space before saccades. Nature, 386(6625), 598-601.

Schiff, W., \& Detwiler, M. L. (1979). Information used in judging impending collision. Perception, 8(6), 647-658. https://doi.org/10. 1068/p080647

Sheth, B. R., \& Shimojo, S. (2001). Compression of space in visual memory. Vision Research, 41(3), 329-341. https://doi.org/10.1016/ S0042-6989(00)00230-3

Watanabe, K. (2004). Visual grouping by motion precedes the relative localization between moving and flashed stimuli. Journal of Experimental Psychology: Human Perception and Performance, 30, 504-512. https://doi.org/10.1037/0096-1523.30.3.504

Watanabe, K., Nijhawan, R., Khurana, B., \& Shimojo, S. (2001). Perceptual organization of moving stimuli modulates the flash-lag effect. Journal of Experimental Psychology: Human Perception and Performance, 27, 879-894. https://doi.org/10.1037/0096-1523.27. 4.879

Watanabe, K., \& Yokoi, K. (2006). Object-based anisotropies in the flashlag effect. Psychological Science, 17(8), 728-735. https://doi.org/ 10.1111/j.1467-9280.2006.01773.x

Whitney, D., \& Cavanagh, P. (2000). Motion distorts visual space: Shifting the perceived position of remote stationary objects. Nature Neuroscience, 3(9), 954-959. https://doi.org/10.1038/78878 